\title{
Clinical significance of HOX11L2 and HOX11 gene expression in T-ALL patients
}

\author{
Said H. Abdu' ${ }^{1}$, Doaa Shahin ${ }^{2}$, Mohamed R. El-Shanshory ${ }^{3}$, Hoda A. Salem ${ }^{4}$, Eman A. Amer ${ }^{5}$, \\ Mohamed M. El-Shebeiny ${ }^{6}$ \\ ${ }^{1}$ Department of Clinical Pathology, Faculty of Medicine, Tanta University, Tanta, Egypt \\ ${ }^{2}$ Department of Clinical Pathology, Faculty of Medicine, Mansoura University, Mansoura, Egypt \\ ${ }^{3}$ Department of Pediatric, Faculty of Medicine, Tanta University, Tanta, Egypt \\ ${ }^{4}$ Department of Clinical Pharmacy, Faculty of pharmacy, Misr University for Science and Technology, Cairo, Egypt \\ ${ }^{5}$ Biochemistry Department, Faculty of pharmacy, Modern Science and Art University, Cairo, Egypt \\ ${ }^{6}$ Department of Clinical Oncology, Faculty of Medicine, Tanta University, Tanta, Egypt
}

Email address:

doaashahin2007@yahoo.com (D. A. Shahin)

\section{To cite this article:}

Said H. Abdu, Doaa Shahin, Mohamed R. El-Shanshory, Hoda A. Salem, Eman A. Amer, Mohamed M. El-Shebeiny. Clinical Significance of HOX11L2 and HOX11 Gene Expression in T-ALL Patients. Journal of Cancer Treatment and Research. Vol. 2, No. 3, 2014, pp. 27-32. doi: 10.11648/j.jctr.20140203.12

\begin{abstract}
Objective: The aim of this study was to determine the prognostic value of two structurally related homeobox genes TLX1/HOX11 and TLX3/HOX11L2 on the clinical outcome of T-ALL patients. Material and Methods: The study included 28 newly diagnosed T-ALL patients. HOX11L2 and HOX11 gene expression were detected by real time PCR. Patients received treatment according to the ALL BFM-90 protocol. Results: Of 28 patients, 8(28.6\%) expressed HOX11L2 and 4(14.3\%) expressed HOX11.The overall survival of patients with HOX11L2 expression was lower than of the patients without HOX11L2 expression (log-rank $\mathrm{P}<0.025)$. As regards HOX11 expression, a statistically significant difference in clinical outcome was found, where HOX11 expression conferred a prognostic advantage $(p<0.001)$. Conclusion: the present study was showed that the outcome of HOX11L2 and HOX11 expression differs, with poor outcome for patients with HOX1112-expression. Future molecular diagnostics may make use of such leukemia-specific markers as HOX11L2 and HOX11 to detect minimal residual disease.
\end{abstract}

Keywords: HOX11L2, HOX11, Real Time PCR, T-ALL

\section{Introduction}

T- cell ALL (T-ALL) accounts for $15 \%$ of all newly diagnosed pediatric ALL cases and is clinically regarded as a high-risk disease with an unfavorable prognosis ${ }^{(1,2)}$. Treatment of acute lymphoblastic leukemia (ALL) has greatly improved over the last decades, leading to cure in approximately $80-85 \%$. Recently with the use of modern risk- adjustment multiagent treatment regimens, the fiveyear event free survival rate became of approximately 70 $75 \%$, but still a significant proportion of patients $(30 \%)$ continue to relapse and they are considered as extremely poor prognosis ${ }^{(3)}$.

A large proportion of cases shows a normal karyotype and recurrent chromosomal abnormalities are detectable only in about $50 \%$ of T-ALL using conventional cytogenetic ${ }^{(4)}$. For most of these abnormalities, the T-cell receptor (TCR)- $\alpha / \delta$ locus $(14 q 11)$ or TCR- $\beta$ locus $(7 q 34)$ come close to target genes and activate their ectopic expression ${ }^{(5,6)}$. The introduction of novel technologies has allowed an increasing number of alterations to be obtained by using molecular biology, FISH and gene expression profiling, which have enabled five subgroups to be recognized, i.e. immature/LYL1, TAL, HOX11, HOX11L2, and $\operatorname{HOX}(7,8,9,10)$. These genes are frequently targeted during the malignant transformation of $\mathrm{T}$ cells by others mechanisms, such as interstitial deletion on chromosome 1 for TAL1/SCL or point mutations for NOTCH1 ${ }^{(11)}$. Other translocations not involving TCR loci leading to the formation of specific fusion genes like CALM-AF10, or MLL rearrangements have also been described ${ }^{(12)}$.

Studies have revealed that certain transcriptional factors associated with T-ALL might be useful for prognosis. The most notable examples are two structurally related 
homeobox genes TLX1/HOX11 and TLX3/HOX11L2 $2^{(13)}$. HOX11 is aberrantly expressed in childhood T-ALL as a result of either two chromosomal translocations, $\mathrm{t}(7 ; 10)(\mathrm{q} 35 ; \mathrm{q} 24)$ and $\mathrm{t}(10 ; 14)(\mathrm{q} 24 ; \mathrm{q} 11)$ which place the HOX11 coding sequence under the transcription control of T-cell receptor regulatory elements. HOX11L2 is involved in a cryptic translocation, $\mathrm{t}(5 ; 14)(\mathrm{q} 35 ; \mathrm{q} 32)$, detected only by FISH resulting in its ectopic transcription ${ }^{(10,14)}$.

The aim of this study was to determine the prognostic value of two structurally related homeobox genes TLX1/HOX11 and TLX3/HOX11L2 on the clinical outcome of T-ALL patients.

\section{Materials and Methods}

This study was conducted on 28 patients with T-ALL, attending the haemato-oncology Unit of Pediatric Department and Clinical Oncology Department at Tanta University Hospitals and Tanta Cancer Center, in the period between February 2002 and December 2006. There were 20 males and 8 females, aged from 3 to 13 years. Ten apparently health children of matched age and sex were included in the study as controls. All patients are subjected to complete clinical examination. Diagnosis was based on complete haemogram, BM examination, haematomorphology and immunophenotyping by expression of cytoplasmic and or surface CD3, CD5, CD7, CD1a and negativity for CD19 and MPO according to the European EGIL recommendation ${ }^{(15)}$.

Diagnostics peripheral blood or B.M. samples were taken from 28 patients with T-ALL. Informed consent was obtained from the patients parents in all cases. Follow up of patients were carried out clinically and by blast count in Bone marrow (BM). The white blood cell count with median 102.5 (range from $6-160 \times 10^{9} / \mathrm{L}$ ), 11 cases with presenting mediastinal mass (39.2\%), 5 cases with Central nervous system ( CNS) involvement ( $17.8 \%), 24$ cases with hepatomegaly $(85.7 \%), 25$ cases with splenomegaly ( $89.2 \%$ ), and 23 cases with lymphadenopathy ( $82.1 \%$ ).

Patients were treated according to the ALL BFM-90 Protocol medium-risk group (MRG). The protocol consists of: Induction, followed by consolidation and reinduction in MRG.The number of leukemic blasts in the blood on day 8 was calculated from the absolute leukocyte count. The presence of at least $1000 / \mu \mathrm{L}$ blasts in $\mathrm{PB}$ on day 8 was defined as a "prednisone: poor response" (PPR) and the presence of $5 \%$ or greater marrow blasts on day 33 , shift the treatment to the high risk protocol ${ }^{(16)}$. In high risk group (HRG), the induction followed by intensive reconsolidation (Element HR1, Element HR2, Element HR3) that were repeated 3 times. CNS-directed preventive therapy for HRG patients consisted of 3 doses of intrathecal MTX in induction, and 9 doses of triple-drug intrathecal therapy (MTX, cytarabine, and prednisolone) during intensive consolidation. Preventive CNS irradiation at a dose of 12 Gy was given only in MRG patients at the end of reinduction and in HRG patients after intensive consolidation. In patients who initially had CNS disease, Cranial radiation therapy (CRT) was administered in ageadapted dosages; thus, patients under 1 year of age received no CRT, patients older than 1 year of age but under 2 years of age received $18 \mathrm{~Gy}$, and patients 2 years of age or older received $24 \mathrm{~Gy}$. These patients also received 2 additional doses of intrathecal MTX in both induction and reinduction, if in the HRG, 1 additional administration of intrathecal triple-drug therapy (MTX, cytarabine, and prednisolone) in each cycle HR-2. In boys with clinically overt testicular involvement, local irradiation (24 Gy) was performed. Other forms of local radiotherapy were not scheduled in protocol ALL-BFM 90. Maintenance therapy was initiated 2 weeks after the end of reinduction or the ninth HR element. The scheduled dose of 6-mercaptopurine was 50 $\mathrm{mg} / \mathrm{m}^{2}$ a day given orally. MTX was given orally at a dose of $20 \mathrm{mg} / \mathrm{m}^{2}$ once a week, with adjustments in dosage made in accordance with the white blood cell (WBC) count For all patients, the total duration of therapy was 24 months.

Samples Collection was as follow: BM Aspirates and venous blood samples were collected from each patient under complete aseptic technique. Ten normal peripheral blood lymphocyte RNA were extracted from healthy donors' samples as control group to determine the reference level of TLX1 and TLX3.

RNA was extracted using RNA isolation kit (QIAamp RNA Blood Mini Kit [Qiagen] Cat. No. 52304 according to manufacturers' instructions. (QIAGEN Inc. Valencia • CA. USA) RNA was reversed transcribed from $1 \mu \mathrm{g}$ total RNA in a final volume of $20 \mu \mathrm{l}$ containing reverse transcription polymerase chain reaction (RT-PCR) buffer $(1 \mathrm{mM}$ each dNTP, $3 \mathrm{mM} \mathrm{MgCl} 2,75 \mathrm{mM} \mathrm{KCl}, 50 \mathrm{mM}$ Tris-HCL pH 8.3) $10 \mathrm{U}$ RNA asin (promega Madison, WI), $100 \mathrm{mM}$ dithiothretol, 100U superscript II (Gibco- BRL, Cergy Pontoise, France), $25 \mu \mathrm{M}$ random hexamers. The expression levels of various target PCR were quantified relative to the expression level of the endogenous housekeeping gene, glyceradhyde-3- phosphate dehydrogenase (GAPDH), by real time RT-PCR in an StepOne 7400 (PE Applied Biosystems, foster city, CA.USA) as described previously ${ }^{(18,19)}$.

For detection of TLX1/HOX11 expression levels, the forward primer 5 '-CTC ACT GGC CTC ACC TT-3' and reverse primer 5 - CTG TGC CAG GCT CTT Ct- 3` were used in combination with the probe $5^{\circ}-(\mathrm{FAM})$ - CCT TCA CAC GCC TGC AGA TC- (TAMRA)-3'. For detection of TLX3/HOX 11L2 expression levels, the forward primer 5'TCT GCG AGC TGG AAA A-3 ' and reverse primer 5'GAT GGA GTC GTT GAG GC-3' were used in combination with probe 5 - (FAM)- CCA AAA CCG GAG GAC CAA GT- (TAMRA)-3`(18,19).

\section{Statistical Analysis}

The data collected were statistically analyzed using statistical package for social sciences (SPSS/version 15) software. (Inc., Chicago, USA). Variables considered were 
patients, age and gender and disease activity and treatment characteristics. All patients still alive in first continuous remission were censored at their last follow-up. Probabilities of overall survival were calculated using the Kaplan-Meier estimate and P- values were determined using the Log-rank test. The Cox model was used to determine the independent prognostic importance of variables. Further analyses were performed only on patients with available molecular data to evaluate the clinical significance of the genetic mutations. Patient-, disease-, and treatment-related variables of the groups divided according to genetic mutations were compared using the $x 2$ statistic for categorical variables and the Mann-Whitney test for continuous variables. The log-rank test and Cox proportional hazards model were used to determine the univariate and independent prognostic importance of several variables.

\section{Results}

Expression of HOX11L2 and HOX11 were studied in 28 pediatric T-ALL patients. They were 19 patients $<9$ years, range from 3-8 years (median 6) and 9 patients $>$ range from 9-13 years (median 10.5). There were 15 male (53.6\%) and 13 female (46.4\%). The clinical characteristics of these patients are summarized in table (1). Of 28 patients, $8(28.6 \%)$ expressed HOX11L2 and 4(14.3\%) expressed HOX11. They were no statistical significance between HOX1112 and HOX11 expression with respect to age, sex $(\mathrm{p}=0.504$ and 0.86 respectively), presenting mediastinal mass $(\mathrm{P}=0.635,0.761)$ and $\mathrm{CNS}$ involvements $(\mathrm{P}=0.538$ and 0.283 respectively). Nevertheless, HOX1112 and HOX11 strongly correlate with hepatomegaly $(\mathrm{P}=0.04$, 0.001), splenomegaly $(\mathrm{P}=0.05, \quad 0.022) \quad$ and lymphadenopathy $(\mathrm{P}=0.05,0.001)$ respectively. White blood cells were significantly higher in HOX11L2 subgroup than HOX $11(\mathrm{p}=0.014)$. The clinical responses to treatment of patients with the two oncogenetic subgroup are detailed in table (1). For each subgroup the relationship with outcome was assessed by Kaplan-Meier curves (figure 1 and 2). The overall survival of patients with HOX11L2 expression was lower than of the patients without HOX11L2 expression (log-rank $\mathrm{P}<0.025)$. As regards HOX11 expression, a statistically significant difference in clinical outcome was found, where HOX11 expression conferred a prognostic advantage $(p<0.001)$. We conducted subgroup analyses for all diagnostic parameters, including WBCs, age and sex, response to prednisone. Non of the groups showed a significant difference except poor prednisone response with HOX11 L2 expression $(\mathrm{P}=0.007)$.

Table (1). Clinical Biological and Molecular ontogenetic analysis of pediatric ALL total number 28.

\begin{tabular}{|c|c|c|c|c|c|c|c|}
\hline & & \multicolumn{2}{|c|}{ HOX11L2 } & \multirow{2}{*}{$\mathbf{P}$} & \multicolumn{2}{|l|}{ HOX11 } & \multirow{2}{*}{$\mathbf{P}$} \\
\hline & & $+\mathrm{ve}(8)$ & -ve (20) & & $+v e(4)$ & -ve (24) & \\
\hline \multicolumn{8}{|c|}{ Median age, years at diagnosis: } \\
\hline \multicolumn{8}{|c|}{$<9$ years $($ no. 19$)$} \\
\hline Median & 6 & 4.5 & 6.5 & 0.504 & 4.5 & 6 & 0.086 \\
\hline Range & $3-8$ & $3-8$ & $3-7$ & & $4-6$ & $3-8$ & \\
\hline \multicolumn{8}{|l|}{$>9$ years $($ no. 9$)$} \\
\hline Median & 11 & 10.5 & 11 & 0.171 & 6 & 11 & 0.102 \\
\hline Range & $9-13$ & $10-11$ & $9-13$ & & $3-8$ & $9-13$ & \\
\hline \multicolumn{8}{|c|}{ Childs gender M/F (15/13): } \\
\hline M & $15(53.6 \%)$ & $7(87.5 \%)$ & $11(55 \%)$ & 0.044 & $3(20 \%)$ & $12(80 \%)$ & 0.628 \\
\hline $\mathrm{F}$ & $13(46.4 \%)$ & $1(12.5 \%)$ & $9(45 \%)$ & 0.235 & $1(7.7 \%)$ & $12(92.3 \%)$ & 0.548 \\
\hline \multicolumn{8}{|l|}{ Liver: } \\
\hline Normal & $4(14.29 \%)$ & $3(37.5 \%)$ & $1(5 \%)$ & 0.620 & $2(50 \%)$ & $2(9 \%)$ & 0.896 \\
\hline Enlarged & $24(85.71 \%)$ & $5(62.5 \%)$ & $19(95 \%)$ & 0.049 & $2(50 \%)$ & $22(91 \%)$ & 0.001 \\
\hline \multicolumn{8}{|l|}{ Spleen : } \\
\hline Normal & $3(10.71 \%)$ & $2(25 \%)$ & $1(5 \%)$ & 0.639 & $2(25 \%)$ & $1(4.2 \%)$ & 0.587 \\
\hline Enlarged & $25(89.29 \%)$ & $6(75 \%) /$ & $19(95 \%)$ & 0.050 & $2(75 \%)$ & $23(95.8 \%)$ & 0.022 \\
\hline \multicolumn{8}{|l|}{ Lymph nodes: } \\
\hline Normal & $5(17.86 \%)$ & $4(50 \%)$ & $1(5 \%)$ & 0.538 & $3(75 \%)$ & $2(9 \%)$ & 0.239 \\
\hline Enlarged & $23(82.14 \%)$ & $4(50 \%)$ & $19(95 \%)$ & 0.05 & $1(25 \%)$ & $22(91 \%)$ & 0.001 \\
\hline $\begin{array}{l}\text { Mediastinal } \\
\text { involvement }\end{array}$ & $11(39.3 \%)$ & $4(50 \%)$ & $7(35 \%)$ & 0.635 & $2(50 \%)$ & $9(37.5 \%)$ & 0.761 \\
\hline CNS involvement & $5(17.9 \%)$ & $1(12.5 \%)$ & $4(20 \%)$ & 0.538 & $2(50 \%)$ & $3(12.5 \%)$ & 0.283 \\
\hline $\begin{array}{l}\text { WBCs median at } \\
\text { diagnosis } 10^{9} / \mathrm{L}\end{array}$ & 102.5 & 115 & 92.5 & \multirow[t]{2}{*}{0.014} & 67 & 90 & \multirow[t]{2}{*}{0.059} \\
\hline Range & $6-160$ & $24-152$ & $6-160$ & & $6-120$ & $6-160$ & \\
\hline \multicolumn{8}{|c|}{ Response to pre-phase treatment: } \\
\hline GPR & $12(42.86 \%)$ & $5(62.5 \%)$ & $7(35 \%)$ & 0.469 & $4(100 \%)$ & $8(33.3 \%)$ & 0.167 \\
\hline PPR & $16(57.14 \%)$ & $3(37.5 \%)$ & $13(65 \%)$ & 0.007 & $0(0 \%)$ & $16(66.6 \%)$ & 0.112 \\
\hline \multicolumn{8}{|l|}{ Failure: } \\
\hline Early deaths & $4(14.29 \%)$ & $2(25 \%)$ & $2(10 \%)$ & 0.099 & $1(25 \%)$ & $3(12.5 \%)$ & 0.365 \\
\hline Toxic death & $6(21.24 \%)$ & $0(0 \%)$ & $6(30 \%)$ & 0.038 & $0(0 \%)$ & $6(25 \%)$ & 0.001 \\
\hline
\end{tabular}

GPR: good prednisone response; PPR: Poor prednisone response, CNS: Central nervous system, BM: Bone Marrow, PB: Peripheral blood. 
Multivariate Cox proportional hazard analyses using WBCS, age, gender and overall survival as variables. Age at diagnosis was given in years, WBCS at diagnosis were given as $10^{9} / \mathrm{L}$ table (2). A poor response to prednisone and over expression of HOX11L2 independently predicted adverse outcome. The hazard ratio for overall survival patients with poor response to prednisone were 2.3 $(\mathrm{P}=0.001)$ and $2.7(\mathrm{P}=0.01)$ for patients with HOX11L2 positive T-ALL.

Table (2). Multivariate analyses using Cox regression model with factors associated with overall survival.

\begin{tabular}{llll}
\hline & Hazard ratio & $\mathbf{9 5 \%}$ CI & P \\
\hline HOX 11L2 & 2.698 & $0.869-1.027$ & 0.012 \\
HOX 11 & 1.986 & $0.563-1.658$ & 0.098 \\
WBC & 1.00 & $0.869-1.028$ & 0.325 \\
Age & 1.032 & $0.934-1.327$ & 0.354 \\
Sex & 0.758 & $0.189-1.365$ & 0.741 \\
Response to steroid & & & \\
Good 3 & 0.573 & $0.475-1.325$ & 0.547 \\
Poor 5 & 2.325 & $1.369-6.71$ & 0.001 \\
\hline
\end{tabular}

Univariate analysis COX proportional hazard analysis using overall survival for the HOX11L2 and HOX11. Hazard ratio for each specific oncogenetic subgroup was indicated relative to T-ALL cases lacking that molecular aberration table (3). Only two factors were significantly correlated with lower overall survival rate: a poor response to prednisone $(\mathrm{P}=0.05)$ and HOX11L2 status $(\mathrm{P}=0.036)$.

Table (3). uinvariate analyses using Cox regression model with factors associated with overall survival

\begin{tabular}{llll}
\hline & Hazard ratio & $\mathbf{9 5 \%}$ CI & P \\
\hline HOX 11L2 & 2.411 & $0.983-5.681$ & 0.036 \\
HOX 11 & 0.271 & $0.698-4.658$ & 0.527 \\
WBC & 0.985 & $0.088-4.058$ & 0.328 \\
Age & 0.677 & $1.396-9.986$ & 0.102 \\
Sex & 0.726 & $0.340-1.625$ & 0.307 \\
Response to steroid & & & \\
Good 3 & 0.963 & $0.478-2.365$ & 0.05 \\
Poor5 & 1.211 & $0.262-6.550$ & \\
\hline
\end{tabular}

Overall survival:

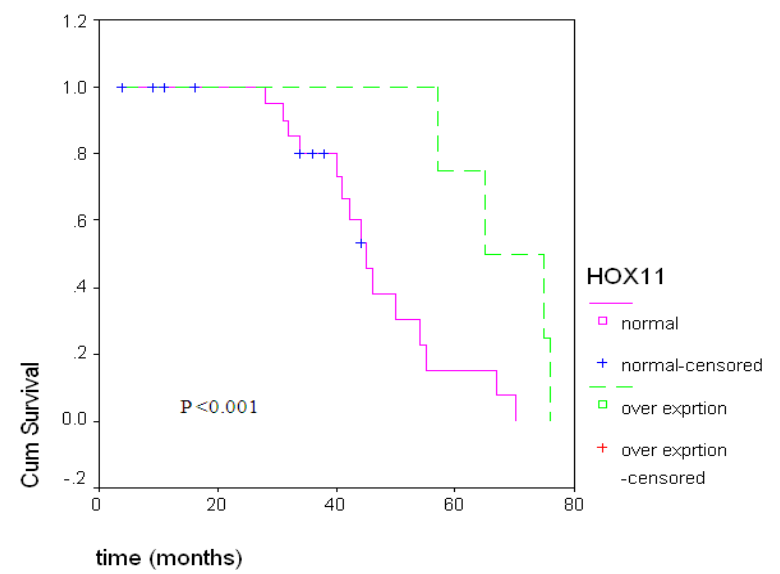

Fig. (1). Clinical outcome of patients with leukemia cells expressing HOX11 positive and negative cases.

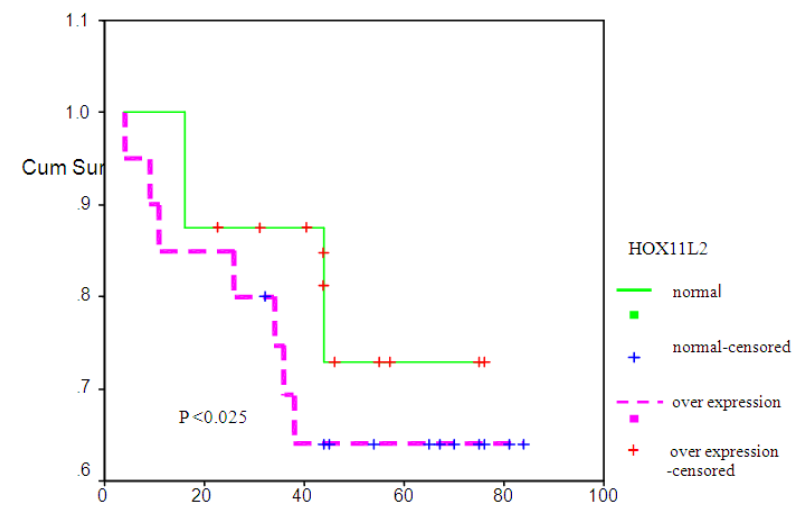

Fig. (2). Clinical outcome of patients with leukemia cells expressing HOX11L2 positive and negative cases.

\section{Discussion}

Treatment optimization in acute leukemia requires accurate assignment of patients at diagnosis to specific risk groups to guide subsequent risk adapted treatment stratification. In T-ALL, few prognostic molecular markers have been identified ${ }^{(17)}$. In this study, we had evaluated the impact of expression of the genes HOX11L2 and HOX11 in conjunctions with other clinical factors to predict outcome in T-ALL patients.

A panel of 28 pediatric patients with T-ALL was investigated for the presence of expression of HOX11L2 and HOX11 genes. The expression of HOX11L2 was found in $8(28.6 \%)$ and HOX11 expression was found in $4(14.3 \%)$ patients, in keeping with previous reports by others ${ }^{(18,19)}$. In contrast, Mauvieux et al ${ }^{(20)}$ was found $60 \%$ of cases expressed HOX11L2 and 5\% in pediatric cases as regards HOX11 expression by Bergeron et al ${ }^{(21)}$..

Bernard et al, ${ }^{(9)}$ initially reported cases of T-ALL with $\mathrm{t}(5 ; 14)(\mathrm{q} 35 ; \mathrm{q} 32)$ and expression of the developmental gene HOX11L2 which belongs to a distinct family of homeobox genes including HOX11, HOX11L1 and HOX11L2. These genes harbor a threonine in the third helix of the homeodomain, which confers specific DNA binding properties ${ }^{(22,23)}$. Ectopic expression of HOX11 in T-ALL is associated with translocations implicating TCR genes $t(10 ; 14)$ and $t(7 ; 10)$ for TCR alpha and TCR beta, respectively ${ }^{(11,23)}$. In $\mathrm{T}$-ALL with $\mathrm{t}(5 ; 14)(\mathrm{q} 35 ; \mathrm{q} 32)$, HOX11L2 expression is probably under the influence of the CTIP2 (BCL11B )gene ${ }^{(9)}$ located on chromosome 14. The latter gene is strongly expressed in the thymus, and is potentially implicated in T cell differentiation. HOX11L2 activation might occur independent of $\mathrm{t}(5 ; 14)$ translocation e.g. microdeletion, submicroscopic inversions lead to transcriptional activation of the gene.

HOX11L2 were significantly express in male than females $(\mathrm{P}=0.044)$ and this in agreement with previous studies done by Fernado $2002^{(24)}$ suggesting a clear correlation with male sex, that remains unexplained. There were no association was observed with age at diagnosis and this was in agreement with Mauvieux et al $2002^{(20)}$. In 
contrast, there was a significant correlation with high white blood cell count $(\mathrm{P}=0.014)$. As regards HOX11 there was no statistical differ between male and female, age and WBCs.

HOX11L2 and HOX11 were statistically significant with hepatomegaly, splenomegaly and lymphadenopathy .On the other hand, Ballerini et al $2002^{(18)}$ failed to find any associations.

In the current study, there was a significant predictor for an association between HOX11L2 and poor outcome. This result was in agreement with some studies ${ }^{(19,24,25)}$; but was on the contrary other studies ${ }^{(26,21)}$ indicating that the prognostic relevance of HOX11L2 could be dependent on the therapy given, associations with ABL1 abnormalities, NOTCH1 mutation status and CD1 positivity ${ }^{(20,27)}$. The high frequency of HOX11L2 deregulation in T-ALL suggests its involvement as a key pathway for leukomogenesis.

The present study showed that patients with leukemia cells expressing HOX11 tended to have better clinical outcome compared to the other patients in the study. This is in accordance with previous studies $(19,28)$.

The difference in the prognosis between HOX11L2 and HOX11 may be due to these subgroups are arrested at slightly different T-cell developmental stages and therefore may have different responses to therapy. The presence of different immunophenotypic markers between HOX11L2 and HOX11 supports this view. HOX 11 rearranged T-ALL is arrested at a strictly early cortical ${ }^{(29,30)}$ or immature-B developmental stage while HOX11L2 - rearranged T-ALL comprises both immature and TCR alpha delta-positive mature cases ${ }^{(31)}$.

In conclusion, the present study was showed that the outcome of HOX11L2 and HOX11 expression differs, with poor outcome for patients with HOX11 L2expression.

Cytogenetic, molecular and immunologic studies should be combined in larger series, in order to confirm the correlation between cytogenetic abnormalities, phenotype and HOX11L2 and HOX11 gene expression and to specify its prognostic significance ${ }^{(32)}$.Future molecular diagnostics may make use of such leukemia-specific markers as HOX11L2 and HOX11 to detect minimal residual disease.

\section{References}

[1] Chiaretti S., and Foa R.: T-cell Acute lymphoblastic leukemia. Haematologica 2009; 94 (2):160-162.

[2] Thiel E., Kranze B.R., Raghavcher A., Bartran C.R., Loffler $H$. et al.: Prethymic phenotype and genotype of pre-T (CD7+/ER-) - cell leukemia and its clinical significance within adult acute lymphoblastic leukemia. Blood 1989; $73: 1247-1258$.

[3] Silverman L.B., Gelber R.D., Dalton V.K., Asselin B.L., Barr R.D. et al.: Improved outcome for children with acute lymphobalstic leukemia: results of Dane-Faber consortium protocol 91-01. Blood 2001; 97, 1211-1218.
[4] Harrison C.T. and Foronil L. : Cytogenetics and molecular genetics of acute lymphoblastic leukemia. Rev Clin Exp Hematol.2002; 3:91-113.

[5] Cauwelier B., Dastugue N., Cools J., Poppe B., Herens C. et al.: Molecular cytogenetics study of 126 unselected T-ALL cases reveals high incidence of Tc beta locus rearrangements and putative new T-cell oncogenes. Leukemia 2006; 20:1238-1244.

[6] Carrol A.J., Crist W.M., Ragab A.H. et al.: The $\mathrm{t}(1 ; 14)(\mathrm{p} 34 ; \mathrm{q} 11)$ is nonrandom and restricted to T-cell acute lymphoblastic leukemia: a Pediatric Oncology Group study. Blood 1990; 76:1220-4.

[7] Speleman F., Cauwelier B., Dastugue N., Cools J. et al.: A new recurrent inversion, $\operatorname{inv}(7)(\mathrm{p} 15 \mathrm{q} 34)$, leads to transcription al activation of HOXA10 and HOXA11 in a subset of T-cell acute lymphoblastic leukemias. Leukemia 2005; 19:358-66.

[8] Hatano M., Roberts C.W., Minden M., Crist W.M. et al.: Deregulation of a homeopox gene HOX11 by the $\mathrm{t}(10 ; 14)$ in T-cell leukemia. Science 1991; 253: 79-82.

[9] Bernard O.A., Busson-Leconiat M., Ballerini P., Mauchauffe $M$. et al.: A new recurrent and specific cryptic translocation, $\mathrm{t}(5 ; 14)(\mathrm{q} 35 ; \mathrm{q} 32)$, is associated with expression of the HOX11L2 gene in $\mathrm{T}$ acute lymphoblastic leukemia. Leukemia 2001; 15:1495-504.

[10] Baldus. C.D, Thibaut .J, Goekbuget N., Stroux A., Schlee C., Mossner M., Burmeister T., Schwartz S., Bloomfield C.D., Hoelzer D., Thiel E. and Hofmann W.K.: Prognostic implications of NOTCH1 and FBXW7 mutations in adult acute T-lymphoblastic leukemia. Haematologica 2009;94:1383-1390

[11] Asnafi V., Radford Weiss L., Dastugue N., Bayle C. et al.: CALM-AFT10 is a common fusion transcript in T-ALL and is specific to the TCR gamma delta lineage. Blood 2003; 102:1000-1006.

[12] Harrisan CJ : Cytogenetics of Pediatric and adolescent acute lymphoblastic leukemias. Br J Haematol 2009; 144(2):147-56.

[13] ChenE, HuangX, ZhengY, Li Y, ChesneyA, Ben-David Y, Yang E, Hough MR: Phosphorylation of HOX11/TLX1 on Threonine-247 during mitosis modulates expression of cyclin B1. Molecular Cancer 2010; 9:246

[14] Sekimizu M, Sunami S, Nakazawa A, Hayashi Y, Okimoto Y, Saito AM, Horibe K, Tsurusawa M, Mori T.:Chromosome abnormalities in advanced stage T-cell lymphoblastic lymphoma of children and adolescents: a report from Japanese Pediatric Leukemia/Lymphoma Study Group (JPLSG) and review of the literature. $\mathrm{Br} \mathrm{J}$ Haematol. 2011;154(5):612-7

[15] Bene M.C., Castoldi G., Krapp W., Ludwig W.D. Matutes E. et al.: Proposals for the immunological classification of acute leukemia. European Group for the immunological characteristics of leukemia (EGIL). Leukemia1995; 9:1783-6.

[16] Schrappe M., Reiter A., Ludwig W., Harbott J., Zimmermann $M$. et al.: Improved outcome in childhood acute lymphoblastic leukemia despite reduced use of anthracyclines and cranial radiotherapy: results of trial ALLBFM 90. Blood 2000; 95(11): 3310-22. 
[17] Baldus C.D., Martus P., Burmeister T., Schwartz S., Gokbuget $N$. et al.: Low ERG and BAALC expression identifies a new subgroup of adult acute T-lymphoblastic leukemia with a highly favorable outcome. J of Clinical Oncology 2007; 25; 24: 3739-3745.

[18] Ballerini P., Blaise A., Busson-Le Coiant M. et al.: HOX11L2 expression defining a clinical subtype of pediatric T-ALL associated with poor prognosis. Blood 2002; 100:991-997.

[19] Martin Val, Grotel J.P.P., Meijerink H., Berna Beverloo et al.: The outcome of molecular- cytogenetic subgroups in pediatric T-cell acute lymphoblastic leukemia retrospective study of patients treated according to DCOG or COALL protocols. Haematologica 2006 ; 91(9)1212-21

[20] Mauvieux L., Leymarie V., Helias C., Perrusson N. et al. : High incidence of HOX11L2 expression in children with TALL. Leukemia 2002; 16(12):2417-22.

[21] Bergeron J., Clappier E., Radford I., Buzyn A., Millien C. et al.: Prognostic and oncogenic relevance of TLX1/HOX11 expression level in T-Alls. Blood 2007; 110(7):2324-30.

[22] Dear T.N., Sanchez-Garcia I., Rabbits T.H. : The HOX11 gene encodes a DNA -binding nuclear transcription factor belonging to a distinct family of homeopox genes. Proc Natl Acad USA 1993; 90: 4431-4435.

[23] Kenndy M.A., Gonzalez-Sarmiento R., Kees U.R., Lampert F. et al.: HOX11, a homeopox-containing T-cell oncogene on human chromosome 10q24. Proc Nat Acad Sci USA 1991; 88:8900-04.

[24] Ferrnado A.A., Neuberg D.S., Hams D.O., Staunton J., Loh M.L. et al. : Gene expression signatures define novel oncogenic pathways in $\mathrm{T}$ cell acute lymphoblastic leukemia. Cancer Cell 2002;1:75-87.

[25] Cave H., Suciu S., Preudhomme C., Popper B., Robert A. et al.: Clinical Significance of HOX11L2 expression linked to $\mathrm{t}(5 ; 14)(\mathrm{q} 35 ; \mathrm{q} 32)$, of HOX11 expression, and of SIL-TAL fusion in childhood T-cell malignancies: Blood 2004; 103:442-50.
[26] Gottardo N.G., Jacoby P.A., Sather H.N., Reaman G.H. et al. Significance of HOX11L2/TLX3 expression in children with T-cell acute lymphoblastic leukemia treated on children's cancer group protocols. Leukemia 2005; 19:17058 .

[27] Berger R., Dastugue N., Busson M., Van Den J., Perot C. et al.: $\mathrm{t}(5 ; 14) / \mathrm{HOX} 11 \mathrm{~L} 2$ positive T-cell acute lymphoblastic leukemia. A collaborative study of the group Francais de Cytogenetique Hematologique (GFCH). Leukemia 2003; $17: 1851-7$.

[28] Ferrnado A.A., Neuberg D.S., Dodge R.K., Paietta E. et al.: Prognostic significance of TLX1 (HOX11) oncogene expression in adults with T-cell acute lymphoblastic leukemia.. Lancet 2004; 363: 535-6.

[29] Ludwig W.D., Harbott J., Bartram C.R., Komische B., Sperling $C$. et al.: Incidence and prognostic significance of immunophenotyping subgroups in childhood acute lymphoblastic leukemia.: Experience of the BFM study 86 . Recent Results Cancer Res 1993; 131:269-82.

[30] Pullen J., Shuster J.J., Link M., Borowitz M., Amylon M. et al.: Significance of commonly used prognostic factors differs for children with $\mathrm{T}$ cell acute lymphocytic leukemia (ALL), as compared to those with B-precursor ALL. A pediatric Oncology Group (POG) study. Leukemia 1999; 13: 1696-707.

[31] Asnafi V., Beljord K., Libura M., Villarese P., Millien C. et al.: Age related phenotypic and oncogeneic differences in Tcell acute lymphoblastic leukemia may reflect thymic atrophy. Blood 2004; 104: 4173-80.

[32] Ballerini P., Landman-Parker J., Cayuela J., Asnafi V., Labopin P. et al.: Impact of genotype on survival of children with T-cell acute lymphoblastic leukemia treated according to the French protocol FRALLE-93: the effect of TLX3/HOX11L2 gene expression on outcome. Haematologica 2008; 93(11):1658-65. 